\title{
Gut microbiota profiles and characterization of cultivable fungal isolates in IBS patients
}

\author{
Piero Sciavilla ${ }^{1}$ - Francesco Strati ${ }^{2}$ - Monica Di Paola ${ }^{3}$ - Monica Modesto ${ }^{1} \cdot$ Francesco Vitali $^{4} \cdot$ Duccio Cavalieri $^{3}$. \\ Gian Maria Prati ${ }^{5}$ Maura Di Vito ${ }^{1} \cdot$ Giovanni Aragona $^{5} \cdot$ Carlotta De Filippo $^{4} \cdot$ Paola Mattarelli $^{1}$ (B)
}

Received: 19 July 2020 / Revised: 25 March 2021 / Accepted: 4 April 2021 / Published online: 10 April 2021

(C) The Author(s) 2021

\begin{abstract}
Studies so far conducted on irritable bowel syndrome (IBS) have been focused mainly on the role of gut bacterial dysbiosis in modulating the intestinal permeability, inflammation, and motility, with consequences on the quality of life. Limited evidences showed a potential involvement of gut fungal communities. Here, the gut bacterial and fungal microbiota of a cohort of IBS patients have been characterized and compared with that of healthy subjects (HS). The IBS microbial community structure differed significantly compared to HS. In particular, we observed an enrichment of bacterial taxa involved in gut inflammation, such as Enterobacteriaceae, Streptococcus, Fusobacteria, Gemella, and Rothia, as well as depletion of health-promoting bacterial genera, such as Roseburia and Faecalibacterium. Gut microbial profiles in IBS patients differed also in accordance with constipation. Sequence analysis of the gut mycobiota showed enrichment of Saccharomycetes in IBS. Culturomics analysis of fungal isolates from feces showed enrichment of Candida spp. displaying from IBS a clonal expansion and a distinct genotypic profiles and different phenotypical features when compared to HS of Candida albicans isolates. Alongside the well-characterized gut bacterial dysbiosis in IBS, this study shed light on a yet poorly explored fungal component of the intestinal ecosystem, the gut mycobiota. Our results showed a differential fungal community in IBS compared to HS, suggesting potential for new insights on the involvement of the gut mycobiota in IBS.
\end{abstract}

\section{Key points}

- Comparison of gut microbiota and mycobiota between IBS and healthy subjects

- Investigation of cultivable fungi in IBS and healthy subjects

- Candida albicans isolates result more virulent in IBS subjects compared to healthy subjects

Keywords Irritable bowel syndrome $\cdot$ Gut microbiota $\cdot$ Gut mycobiota

Piero Sciavilla, Francesco Strati and Carlotta De Filippo contributed equally to this work.

Paola Mattarelli

paola.mattarelli@unibo.it

1 Department of Agriculture and Food Sciences, University of Bologna, Viale Fanin 44, 40127 Bologna, Italy

2 Laboratory of Mucosal Immunology, Department of Experimental Oncology, European Institute of Oncology, Via Adamello 16, 20139 Milan, Italy

3 Department of Biology, University of Florence, Via Madonna del Piano 6, 50019, Sesto Fiorentino, Florence, Italy

4 Institute of Agriculture Biology and Biotechnology, National Research Council (CNR), Via Moruzzi 1, 56124 Pisa, Italy

5 Department of Gastroenterology and Hepatology, "G da Saliceto" Hospital, Via Taverna 49, 29121 Piacenza, Italy

\section{Introduction}

Irritable bowel syndrome (IBS) is a highly prevalent, stressrelated functional gastrointestinal (GI) disorder that exhibits different GI and neurological symptoms, such as abdominal pain, diarrhea, constipation, and mood disorders (Ishihara et al. 2013). The gut microbiota plays an important role in modulating the communication between the central nervous system (CNS) and the GI tract, the so-called gut-brain axis (Sinagra et al. 2020). IBS is a clear example of a pathological condition involving alterations of this fine equilibrium between the gut microbiota and the CNS. The involvement of the gut microbiota in IBS physiopathology (Pinto-Sanchez et al. 2017) is suggested by the observation that probiotic treatments of IBS patients result in the relief of both GI and 
neurological symptoms. Furthermore, the gut barrier dysfunction widely demonstrated in IBS patients is strictly linked to microbial dysbiosis, also following into an altered modulation of the host immune system.

The IBS gut microbiota has been extensively studied. Antimicrobial peptides and some Toll-like receptors involved in bacterial recognition were shown to be upregulated in IBS (Ishihara et al. 2013; Simrén et al. 2013; Eisenstein 2016), and gut bacterial dysbiosis was investigated as a peripheral trigger. It has been shown that Faecalibacterium prausnitzii and Bifidobacterium genus were depleted in patients with IBS (Pittayanon et al. 2019).

On the other hand, fungi are still under investigation in IBS. Despite being a minor component of the gut microbial community, evidences indicated that fungi can play a role in gut diseases, as demonstrated in inflammatory bowel disease (Iliev et al. 2012; Richard et al. 2015; Sokol et al. 2017; Limon et al. 2017; Di Paola et al. 2020). Recent evidences, for example, suggested that fungi may partially act in the genesis of visceral hypersensitivity by activating the Dectin- 1 receptormediated Syk/CARD9 signaling pathway (Chi et al. 2020).

Here, we characterized the gut microbiota (both bacterial and fungal communities) in IBS patients, taking into account also the two most common gastrointestinal symptoms related to gut motility, constipation, and diarrhea. Furthermore, we investigated the cultivable component of the gut mycobiota by characterizing the fungal isolates for their genotype and phenotype associated with GI tract resistance and potential virulence traits.

\section{Material and methods}

\section{Subject recruitment}

A total of 20 patients ( 13 males and 7 females, mean age 46.4 \pm 6.3 years, range $37-60$ years) with clinical diagnosis of IBS and 18 sex-matched healthy subjects (HS), as controls (10 males and 8 females, mean age $45.4 \pm 5.8$ years, range 18 50 years) were enrolled to the study (Supplementary Table S1).

The study protocol was approved by the Institutional Review Board of the Department of Gastroenterology, Piacenza Hospital (Italy). All participants, recruited at clinical study sites in Piacenza (Department of Gastroenterology, Hospital of Piacenza, Italy), signed informed consent. Inclusion criteria for IBS patient enrollment were in accordance with the Rome III criteria (Longstreth et al. 2006), providing standard conditions for diagnosis of IBS within a 12week period, such as the presence of recurrent abdominal pain or discomfort at least 3 days/months in the last 3 months (12 weeks), associated with $\geq 2$ of the following criteria: (i) improvement with defecation, (ii) onset associated with a change in stool frequency, (iii) onset associated with a change in stool form (appearance). The criteria are fulfilled with symptoms onset 6 months prior to diagnosis. All the subjects followed a Mediterranean-based diet and they did not take antibiotics or probiotics in the 3 months prior to sample collection.

\section{Fecal sample collection and total genomic DNA extraction}

Fecal samples were collected at one time point, and subjects were carefully instructed on the method for sampling. Stool samples were stored at $4{ }^{\circ} \mathrm{C}$ until refrigerated transport (within $2 \mathrm{~h}$ ) to the laboratory. An aliquot was used for fungal cultivation, while another one was used for bacterial and fungal genomic DNA extraction, then stored at $-80{ }^{\circ} \mathrm{C}$.

Total DNA extraction from fecal samples $(250 \mathrm{mg}$, wet weight) was performed using the FastDNA ${ }^{\mathrm{TM}}$ SPIN Kit for Feces (MP Biomedicals, Santa Ana, CA, USA), following the manufacturer's instructions.

\section{Amplified ribosomal DNA restriction analysis}

From each fecal sample (IBS and HS), bacterial 16S rDNA was amplified by PCR. DNA amplification was carried out in $25 \mu \mathrm{l} \mathrm{PCR}$ mixture containing $1 \times$ of PCR buffer, $2 \mathrm{mM}$ of $\mathrm{MgCl}_{2}, 200 \mu \mathrm{M}$ of dNTPs, $0.4 \mu \mathrm{M}$ of the primer $8 \mathrm{~F}\left(5^{\prime}-\right.$ AGAGTTTGATCCTGGCTCAG-3') and 1510R (5'-CGGT TACCTTGTTACGACTT-3'), 2.5U of Taq polymerase, and $10 \mathrm{ng}$ of gDNA as template. PCR amplicons $(10 \mu \mathrm{l})$ were digested with the HaeIII, AluI, RsaI, and MspI restriction enzymes $(0.5 \mathrm{U} / \mu \mathrm{l}$; Thermo Fisher Scientific, Milan, Italy) at 37 ${ }^{\circ} \mathrm{C}$ for $4 \mathrm{~h}$. Restriction products were separated by electrophoresis, using $2.5 \%$ of agarose gel in $1 \times$ TAE buffer at $80 \mathrm{~V}$ for $3 \mathrm{~h}$ and visualized with $0.5-\mu \mathrm{g} / \mathrm{ml}$ ethidium bromide staining. A 1-kb ladder (GeneRuler $1 \mathrm{~kb}$ DNA ladder, Thermo Fisher Scientific, Milan Italy) was used as a DNA marker.

In order to compare the restriction patterns of each fecal sample, a binary matrix was constructed by using 0 and 1 values, as a function of the absence and presence of fragments in each restriction profile, respectively. Such matrix has been used for the construction of a dendrogram, based on amplified ribosomal DNA restriction analysis (ARDRA) patterns, by calculation of samples' distance similarity according to the Jaccard Index (Levandowsly and Winter 1971) by mean of the "vegdist" function within the vegan R package (Oksanen et al. 2020). Hierarchical clustering according to the UPGMA method was performed by using the hclust function within the stats R package. Furthermore, unrooted neighbor-joining phylogenetic trees have been obtained by using the software package PHYLIP (Phylogeny Inference Package) (Plotree and Plotgram 1989). 
$16 S$ rRNA gene and internal transcribed spacer 1 amplicon preparation, Illumina sequencing, and data analysis

Primer sets specific for the V3-V5 hypervariable regions (Fw: 5'TCGTCGGCAGCGTCAGATGTGTATAAGAGACA GCCTACGGGNGGCWGCAG; Rev: 5'GTCTCGTG GGCTCGGAGATGTGTATAAGAGACAGGACT ACHVGGGTATCTAATCC3') and for internal transcribed spacer 1 (ITS1), corresponding to ITS1F (nt 1761-nt 1779) 5'TCCGTAGGTGAACCTGCGG3' and ITS4R (nt 2390nt2409) 5'TCCTCCGCTTATTGATATGC3', were used for amplification of the bacterial 16S rDNA gene and the fungal ITS1 rDNA region, respectively.

The gene amplicons were then purified and pair-end sequenced on Illumina MiSeq platform (IGA Technology Services, Udine, Italy) using a $2 \times 300$ nucleotide-paired reads protocol.

The raw fastq files were submitted to the European Nucleotide Archive with accession number PRJEB38069 (http://www.ebi.ac.uk/ena/data/view/ PRJEB38069) for 16S rDNA and with accession number PRJEB42596 for ITS1.

Reads were pre-processed using the MICCA pipeline (v. 1.5) (http://www.micca.org) (Albanese et al. 2015). Forward and reverse primer trimming and quality filtering were performed using micca trim and micca filter, respectively. De novo greedy clustering and chimera filtering were performed by using micca otu: operational taxonomic units (OTUs) were assigned by clustering the sequences with a threshold of $97 \%$ pairwise identity, and their representative sequences were taxonomically classified using micca classify with the RDP classifier v 2.11 on $16 \mathrm{~S}$ rRNA gene sequencing data (Wang et al. 2007) and against the UNITE fungal ITS database (Kõljalg et al. 2013) on fungal ITS data. Multiple sequence alignment (MSA) of 16S rDNA sequences was performed using the Nearest Alignment Space Termination (NAST) (DeSantis et al. 2006) algorithm implemented in micca msa with the template alignment clustered at $97 \%$ similarity of the Greengenes database (DeSantis et al. 2006) (release 13_05). For fungal ITS1 sequences, de novo MSA was performed using MUSCLE (Edgar 2004). The phylogenetic trees were inferred using micca tree (Price et al. 2010). Sampling heterogeneity was reduced rarefying samples at the depth of the less abundant sample using micca tablerare. Before rarefaction, two samples with less than 2000 reads from the ITS1 dataset have been removed. Alpha (within-sample richness) and betadiversity (between-sample dissimilarity) estimates were computed using the phyloseq R package (McMurdie and Holmes 2013). Permutational analysis of variance (PERMANOVA) test was performed using the adonis() function in the R package vegan with 999 permutations. Linear discriminant effect size analysis (LEfSe) was performed to find features (microbial taxa) most likely to explain differences between classes
(Segata et al. 2011). All statistical analyses were performed using R ( $\mathrm{R}$ CoreTeam 2012); $p$-values were false discovery rate (FDR)-corrected and considered significant with $p<0.05$ (Benjamini and Hochberg 1995).

\section{Characterization of cultivable fungal isolates from fecal samples}

Isolation and identification of fungal cultivable single-cell pure colonies from stool sample of the two cohorts of IBS patients and HS were performed as previously described by Strati et al. (2016).

\section{Phenotypical characterization of fungal isolates}

Phenotypical traits that could be related to the ability of fungal isolates to survive/colonize the human gut have been studied. Growth at supra-optimal temperatures, $\mathrm{pH}$ impact on growth, resistance to bile acids, invasive growth, and hyphal formation have been tested for each fungal isolates, according to the methods described by Strati et al. (2016b).

Biofilm test was performed on YPD (Qvirist et al. 2016) on a flat-bottom 96-well plate by adding $200 \mu \mathrm{l}$ of a fungal cell suspension equal to $\sim 10^{5}$ colony forming unit $(\mathrm{CFU}) / \mathrm{mL}$ in each well. The plate was incubated at $37{ }^{\circ} \mathrm{C}$ for $48 \mathrm{~h}$. After the incubation period, cell suspensions were aspirated and each well with the adhered fungal cells was washed three times with deionized $\mathrm{H}_{2} \mathrm{O}$ and one time with PBS $1 \times$. Biofilmcoated wells were then incubated with $0.01 \%$ of crystal violet (Sigma-Aldrich, Milan, Italy) for $30 \mathrm{~min}$ and washed as above. Finally, each well of the dried microtiter plate was incubated with $100 \mu \mathrm{l}$ of $100 \% \mathrm{EtOH}$ for $10 \mathrm{~min}$ and biofilms quantified by optical density measurement at $570 \mathrm{~nm}$ with a microplate reader (Synergy2BioTek, Winooski, VT, USA).

\section{Antifungal susceptibility testing}

Candida isolates were tested for the susceptibility to the antifungal fluconazole and 5-flucytosine by measuring minimal inhibitory concentration (MIC), according to the Clinical and Laboratory Standards Institute (CLSI) (CLSI 2017). Antifungal resistance was analyzed according to CLSI clinical breakpoints (Pfaller and Diekema 2012; Castanheira et al. 2014).

\section{Random amplification of polymorphic DNA fingerprinting and phylogenetic analysis}

Candida albicans isolates were genotyped by random amplification of polymorphic DNA (RAPD), using the primer Oligo-2 (5'-TCACGATGCA-3') as previously described (Binelli et al. 2006). PCR conditions were $5 \mathrm{~min}$ at $94{ }^{\circ} \mathrm{C}$, $30 \mathrm{~s}$ at $94{ }^{\circ} \mathrm{C}$ and $30 \mathrm{~s}$ at $36^{\circ} \mathrm{C}$ for 40 cycles, and $2 \mathrm{~min}$ at 
$72^{\circ} \mathrm{C}$, followed by a final extension of $10 \mathrm{~min}$ at $72^{\circ} \mathrm{C}$. DNA amplification was carried out in $25 \mu \mathrm{l}$ PCR mixture containing $1 \times$ PCR buffer, $2 \mathrm{mM} \mathrm{MgCl}_{2}, 200 \mu \mathrm{M}$ of dNTPs, $0.4 \mu \mathrm{M}$ of primer $2.5 \mathrm{U}$ of $\mathrm{Taq}$ polymerase, and $10 \mathrm{ng}$ of gDNA as template. Amplification products were separated using a $1.5 \%$ of agarose gel in $1 \times$ TAE buffer at $80 \mathrm{~V}$ for $2 \mathrm{~h}$ and visualized with $0.5-\mu \mathrm{g} / \mathrm{ml}$ ethidium bromide staining. A $100-5000-\mathrm{bp}$ ladder (GeneRuler ${ }^{\mathrm{TM}}$ Express DNA Ladder, Thermo Fisher Scientific, Milan, Italy) was used as a DNA marker.

A binary matrix was constructed by using 0 and 1 values, as a function of the absence and presence of fragments in each position of the gel for each RAPD profile. Such matrix has been used for the calculation of samples' distance similarity according to the Jaccard Index (Levandowsly and Winter 1971) by mean of the "vegdist" function within the vegan $R$ package and clustered hierarchically according to the UPGMA method by using the hclust function within the stats $\mathrm{R}$ package. Furthermore, unrooted neighbor-joining phylogenetic trees have been obtained by using the software package PHYLIP (Phylogeny Inference Package) (Plotree and Plotgram 1989).

\section{Statistical analysis}

Wilcoxon rank-sum tests and Spearman's correlations were performed using the R software (R CoreTeam 2012) through the stats R package (version 3.1.2) and the psych R package, respectively. All $p$-values have been corrected for multiple hypothesis testing controlling the false discovery rate (Benjamini and Hochberg 1995). Statistical significance of hematological parameters between the two cohorts was determined by Student's $T$ test $(p$-value $<0.05)$.

\section{Results}

\section{The IBS gut microbiota showed dysbiotic profiles and bacterial markers associated with constipation}

In order to explore the gut microbiota composition in IBS, we performed preliminary analyses based on hierarchical clustering of restriction profiles, obtained by amplified ribosomal DNA restriction analysis (ARDRA) on total DNA extracted from each fecal sample. ARDRA profiles showed a different cluster distribution of the IBS samples compared to HS (Supplementary Fig. S1A and S1B).

Therefore, we performed a metataxonomic analysis of bacterial microbiota to better characterize the microbial community structure associated with IBS. Analysis of alpha diversity showed a significant reduction of bacterial richness in IBS compared to HS (Fig. 1a). The microbial community structure, as measured by beta-diversity analysis, was significantly different among IBS and HS (PERMANOVA, $p<0.01$; Fig. 1b). All the hematological parameters (Supplementary Table S1), including ESR that was significantly higher in IBS group than HS (Student's $T$ test, $p$ value $<0.01$ ), as well as other variables (i.e., gender, symptoms etc.), did not affect the overall composition of the gut bacterial and fungal communities (PERMANOVA, $p>0.05$ ).

The taxonomic analysis showed enrichment and depletion of several bacterial taxa (Wilcoxon rank-sum test FDR $p<$ 0.05 , Fig. 1c), as further confirmed by LEfSe (linear discriminant analysis effect size) analysis $(p<0.05, \mathrm{LDA}>|2|$; Supplementary Fig. S2). Unlike previous findings (Clemente et al. 2012), we did not observe differences in the Firmicutes to Bacteroidetes ratio between IBS and HS (Wilcoxon ranksum test, $p=0.82$ ). We found a significant enrichment of Actinomyces, Rothia, Gemella, Streptococcus, Enterococcus, Granulicatella, Fusobacterium, Escherichia/Shigella, Veillonella, and Clostridium cluster XI, and depletion of Anaerostipes, Fusicatenibacter, Odoribacter, Oscillibacter, Roseburia, and Faecalibacterium in IBS compared to HS (Fig. 1c, Supplementary Fig. S2).

Furthermore, we evaluated whether two common GI symptoms in IBS, diarrhea, and constipation, differentially contribute to the gut microbiota composition in IBS patients. We observed a significant enrichment of Fusobacteria, Clostridium cluster XVIII, and Gemella in constipated IBS patients (LEfSe, $p<0.05$, LDA $>|2|$; Supplementary Fig. S3).

\section{Analysis of the gut mycobiota in IBS patients shows enrichment of Saccharomycetes and an overgrowth of $C$. albicans isolates}

Exploration analyses of alpha and beta-diversity of the gut mycobiota showed a decrease of the observed fungal OTUs in IBS compared to HS (Fig. 2a, $p$ value $=0.01$ ) and a significant separation of IBS and HS samples in PCoA ordination based on Bray-Curtis distances (Fig. 2b, PERMANOVA, $p$ value $=0.001)$. In IBS group, constipation and diarrhea did not affect the samples' distribution. Metataxonomic analysis of the fungal communities showed an enrichment of Saccharomycetes in IBS (Fig. 2c, FDR-corrected $p<0.05$, Welch $t$ test).

We further investigated the gut mycobiota composition of IBS and HS groups by characterizing the cultivable fungal isolates from fecal samples. We observed a significantly higher number of cultivable fungi (Fig. 3a, $p=0.028$, Wilcoxon rank-sum test), as well as a trend of increase of fungal species richness ( $p=0.07$, Wilcoxon rank-sum test; Fig. 3b), in IBS compared to HS.

In both cohorts, $C$. albicans was the predominant isolated species, representing the $92.7 \%$ and the $80.5 \%$ of the fungal population in IBS and HS, respectively. Candida glabrata was the second most abundant species isolated in IBS, followed by Galactomyces. On the other hand, the gut mycobiota of 
Fig. 1 Characterization of the bacterial community structure in IBS patients and HS. a Alpha diversity, as measured by Shannon index; $* p<0.05$, Wilcoxon rank-sum test. b Betadiversity analysis performed with PCoA ordination based on the weighted UniFrac distances. c Relative abundances of the significantly different bacterial genera between IBS and HS (Wilcoxon rank-sum test; FDRcorrected $p<0.05$ ) a

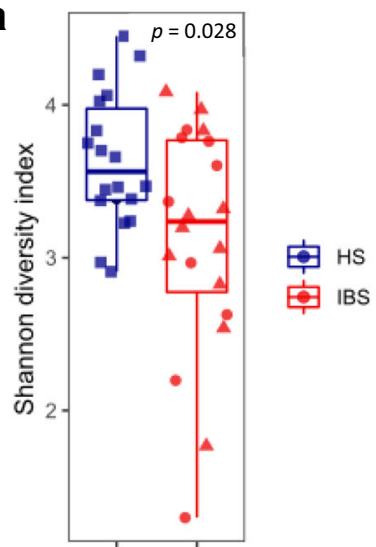

b

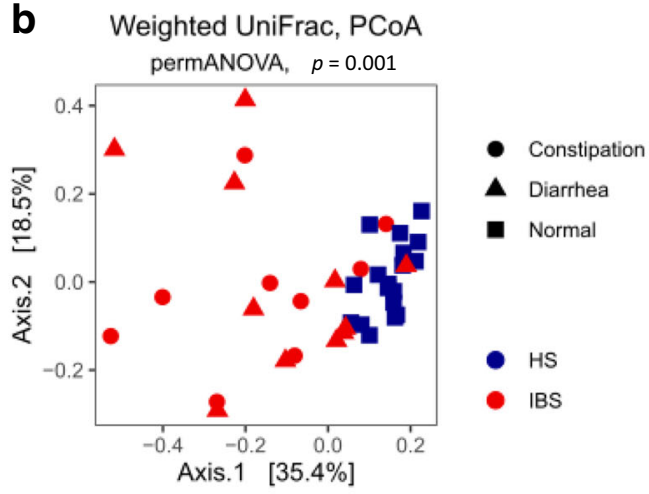

C

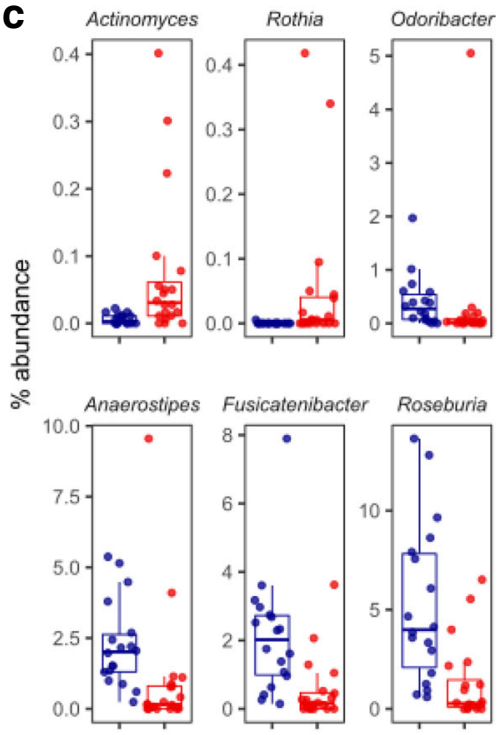

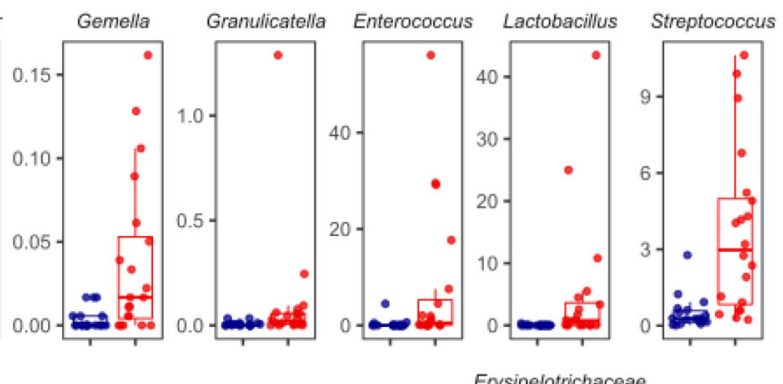

Erysipelotrichaceae
Faecalibacterium Oscillibacter Clostridiales uncl. unclassified Fusobacterium
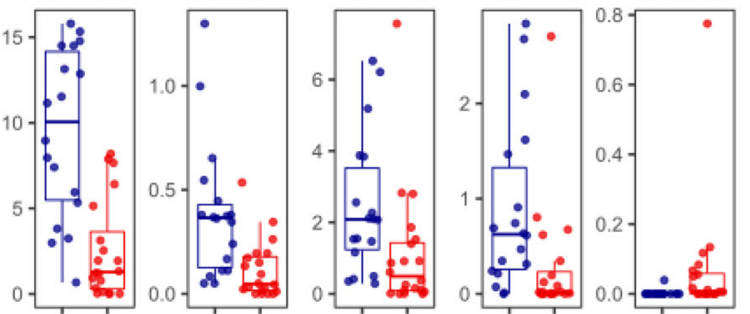

闹 HS
HS group showed a greater diversity compared to IBS, with Candida parapsilosis being the second most represented fungal species in HS, in accordance with findings of Forbes et al. (2019). Furthermore, we found Aspergillus, Rhodotorula, Penicillium, and Pichia genera, known as commensals of diverse body sites (Rizzetto et al. 2014), and fungal species commonly used in food fermentation, such as Torulospora delbrueckii and Starmerella bacillaris (Bely et al. 2008; Englezos et al. 2015), exclusively in HS (Fig. 3c).

\section{C. albicans isolates from IBS are genetically and phenotypically distinct from HS isolates}

The aforementioned results indicated C. albicans as the most abundant species isolated from both IBS and HS. Therefore, we investigated the genetic diversity among these fungal isolates. RAPD genotyping (see "Materials and methods") of C. albicans isolates clearly showed that IBS isolates are genetically unrelated to HS isolates. It is worth to note a clonal expansion of $C$. albicans isolates within some IBS patient (Fig. 4).

Fungi can be passengers, commensals, or transient colonizers of the human GI tract (Rizzetto et al. 2014; Strati et al. 2016; Huseyin et al. 2017). Since stressful conditions (e.g., such as temperature, $\mathrm{pH}$, and presence of bile acids) in the gut ecosystem can influence the microbial community status (Wang et al. 2014), we tested the ability of fungal isolates to resist under GI-like conditions or extreme stress in vitro. We found that $C$. albicans isolates from IBS showed lower growth in respect to HS isolates at the following growth conditions: at $\mathrm{pH} 3$, in the presence of bile acids $(0.5 \%, 1 \%$, and $2 \%$ ), and at supra-optimal temperatures $\left(37^{\circ} \mathrm{C}, 40{ }^{\circ} \mathrm{C}\right.$, and $42^{\circ} \mathrm{C}$ ). On the contrary, C. albicans isolates from IBS patients showed higher growth in respect to HS isolates at the following growth conditions: at $\mathrm{pH} 2$ and at supra-optimal temperatures $\left(44{ }^{\circ} \mathrm{C}\right.$ and $\left.46{ }^{\circ} \mathrm{C}\right)(p<0.05$; Fig. 5a). Furthermore, we tested virulence-related traits, such as the ability to produce hyphae (Fig. 5b) and invade the agar (Fig. 5c). We observed 
Fig. 2 Characterization of the fungal community structure in IBS patients and HS. a Alpha diversity, as measured with observed number of OTUs; * $p<$ 0.05 , Wilcoxon rank-sum test. $\mathbf{b}$ Beta-diversity analysis performed with PCoA ordination based on the Bray-Curtis dissimilarity. c Welch's $t$ test statistics of the relative abundances of the gut mycobiota at family level in IBS (right) and HS (left) subjects. Orange bars indicate significant FDR-corrected $p$ values adjusted for multiple comparison controlling the family-wise type I error rate
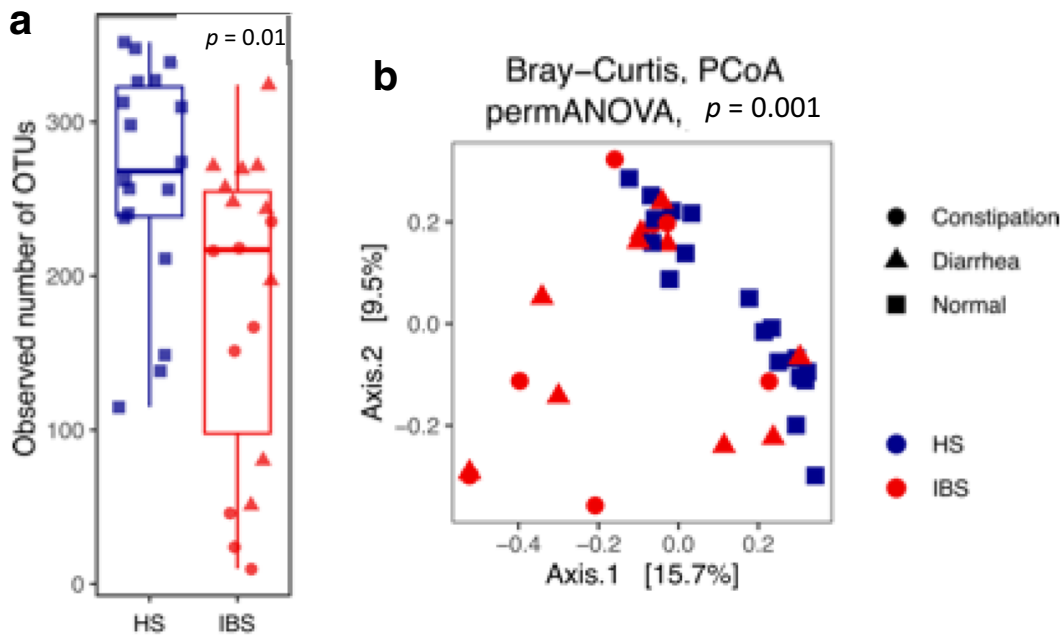

C

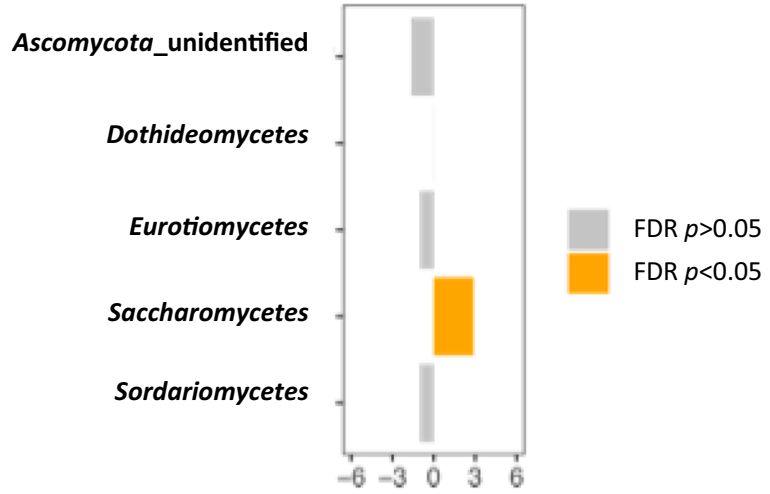

that most $C$. albicans isolates from IBS were able to produce hyphae $(82.1 \%)$, while most $C$. albicans isolates form HS (45.7\%) were not. Moreover, we observed that $C$. albicans isolates able to produce hyphae were more invasive than C. albicans isolates unable to produce hyphae in vitro (FDR-corrected $p<0.01$, Wilcoxon rank-sum test) (Fig. 5d).
IBS and HS subjects' isolates, both able and unable to produce hyphae, were tested for the ability to form biofilm. We did not find significant differences among isolates of the two cohorts (Supplementary Fig. S4). Finally, we compared the susceptibility of $C$. albicans isolates to the most common antifungals, such as fluconazole and the non-azole antifungal 5-flucytosine
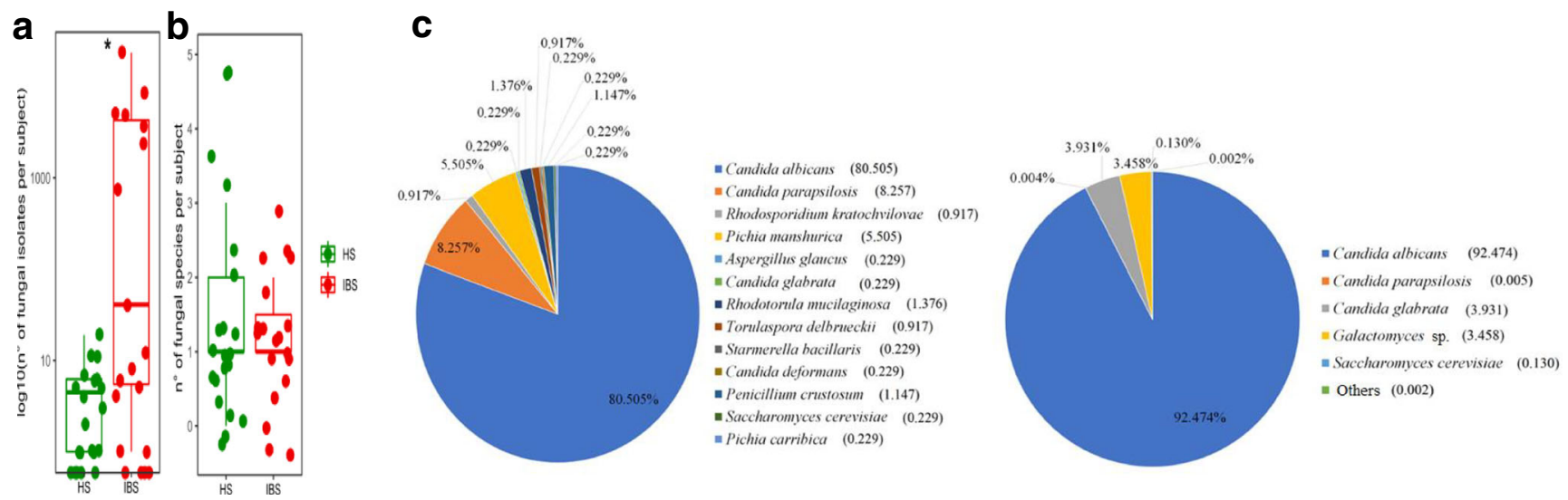

Fig. 3 Cultivable gut mycobiota in IBS and HS groups. a Abundance ( $\log 10$ number of isolates) and b richness of fungal species isolated from stool samples in HS and IBS groups $(* p<0.05$, Wilcoxon rank-sum test). $\mathbf{c}$ Pie charts of the percentage of fungal species identified in (left) HS and (right) IBS 
Fig. 4 Cladogram representation of hierarchal clustering of C. albicans isolates from IBS and HS, based on RAPD profiles. Hierarchical clustering was obtained on calculated Jaccard distances by using UPGMA method. Unrooted neighborjoining phylogenetic trees were obtained by the PHYLIP software package. C. albicans isolates from HS and IBS subjects were colored in green and red, respectively.

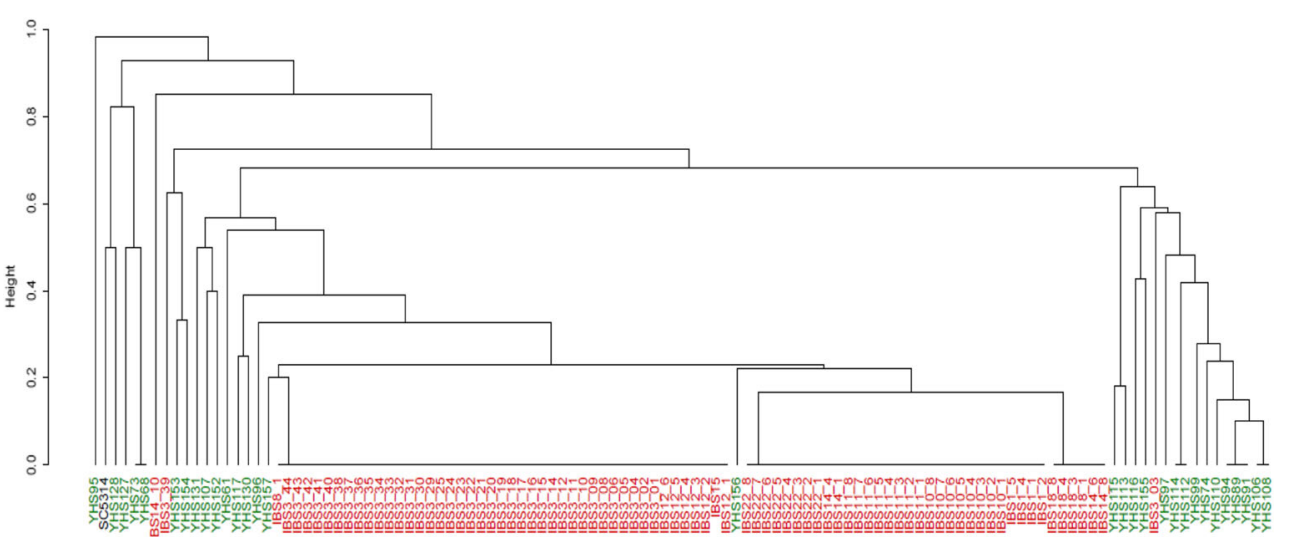

(Table 1). As indicated by MIC90 values, C. albicans isolates from IBS were more susceptible to fluconazole than HS isolates.

\section{Discussion}

The gut microbiota composition and its role in IBS pathophysiology have received great interest from the scientific community (Drossman 2016; Putignani et al. 2016). Its potential involvement in barrier dysfunction and visceral hypersensitivity is now widely recognized (Ishihara et al. 2013; Simrén et al. 2013; Piche 2014; Eisenstein 2016). The presence of bacterial dysbiosis associated to IBS has been confirmed by different metataxonomic studies (Collins 2014; Bhattarai et al. 2017; Li et al. 2019). A higher abundance of Enterobacteriaceae has been found in IBS patients when compared to healthy controls. Bacteria belonging to Enterobacteriaceae family, having the potential ability to invade intestinal epithelial cells or able to exacerbate gut inflammation, were observed in association with inflamed gut mucosa (Øyri et al. 2015).

The role of gut mycobiota in IBS was largely ignored so far. There are evidences about its involvement in colitis
(Iliev et al. 2012), in particular in IBD (Sokol et al. 2017; Di Paola et al. 2020) and, not last, in stress-induced visceral hypersensitivity (Botschuijver et al. 2017). It has also been reported that some metabolic products of yeast metabolism could lead to symptoms related to IBS, and that fungal $\beta$ glucans of the cell wall that are normally recognized by the host immune system can induce visceral hypersensitivity (Botschuijver et al. 2017; Gayathri et al. 2020) and differential immune responses (Rizzetto et al. 2016; Di Paola et al. 2020).

In the present work, the explorative characterization of the intestinal microbial community structure by the ARDRA confirmed that the gut microbiota of IBS patients differs from HS, as previously described (Carroll et al. 2012; Vich Vila et al. 2018). Moreover, metataxonomic analysis showed a depletion of bacterial genera characterized by anti-inflammatory properties, such as Roseburia and Faecalibacterium and enrichment of well-known bacteria found involved in chronic gut inflammation, such as Fusobacterium (Bashir et al. 2016). The differential bacterial community structure in IBS compared with healthy controls, as observed in the present study, was in accordance with previous evidences (Tap et al. 2017).

Table 1 Antifungal resistance of Candida isolates from HS or IBS subjects

\begin{tabular}{|c|c|c|c|c|c|c|c|c|c|c|c|}
\hline \multirow[t]{3}{*}{ Species (no. of isolates HS-IBS) } & \multirow[t]{3}{*}{ Antifungals } & \multicolumn{5}{|c|}{ Healthy subjects (HS) } & \multicolumn{5}{|c|}{ IBS subjects } \\
\hline & & \multicolumn{10}{|c|}{ MIC $(\mu \mathrm{g} / \mathrm{ml})$ clinical breakpoints } \\
\hline & & $\mathrm{MIC}_{50}$ & $\mathrm{MIC}_{90}$ & $\% \mathrm{~S}$ & $\% \mathrm{SDD}$ & $\% \mathrm{R}$ & $\mathrm{MIC}_{50}$ & $\mathrm{MIC}_{90}$ & $\% \mathrm{~S}$ & $\% \mathrm{SDD}$ & $\% \mathrm{R}$ \\
\hline \multirow[t]{2}{*}{ Candida albicans (35-83) } & Fluconazole & 0.5 & $>64$ & 71.4 & 0 & 28.6 & 0.125 & 32 & 86.8 & 2.4 & 10.8 \\
\hline & 5-Flucytosine & 0.125 & 0.5 & 94.3 & 0 & 5.7 & 0.125 & 0.125 & 100 & 0 & 0 \\
\hline \multirow[t]{2}{*}{ Candida parapsilosis (6-6) } & Fluconazole & 0.125 & 0.5 & 100 & 0 & 0 & 0.125 & 0.125 & 100 & 0 & 0 \\
\hline & 5-Flucytosine & 0.125 & 0.125 & 100 & 0 & 0 & 0.125 & 0.125 & 100 & 0 & 0 \\
\hline \multirow[t]{2}{*}{ Candida glabrata $(1-4)$} & Fluconazole & 0.125 & 0.125 & 0 & 100 & 0 & 32 & 32 & 0 & 100 & 0 \\
\hline & 5-Flucytosine & 0.125 & 0.125 & 100 & 0 & 0 & 0.125 & 0.125 & 100 & 0 & 0 \\
\hline
\end{tabular}

$S$, susceptible; $S D D$, susceptible dose-dependent or intermediate; $R$, resistant; $M I C$, minimal inhibitory concentration ranges: fluconazole, $0.125-64$ $\mu \mathrm{g} / \mathrm{ml} ; 5$-flucytosine, $0.125-64 \mu \mathrm{g} / \mathrm{ml}$ 


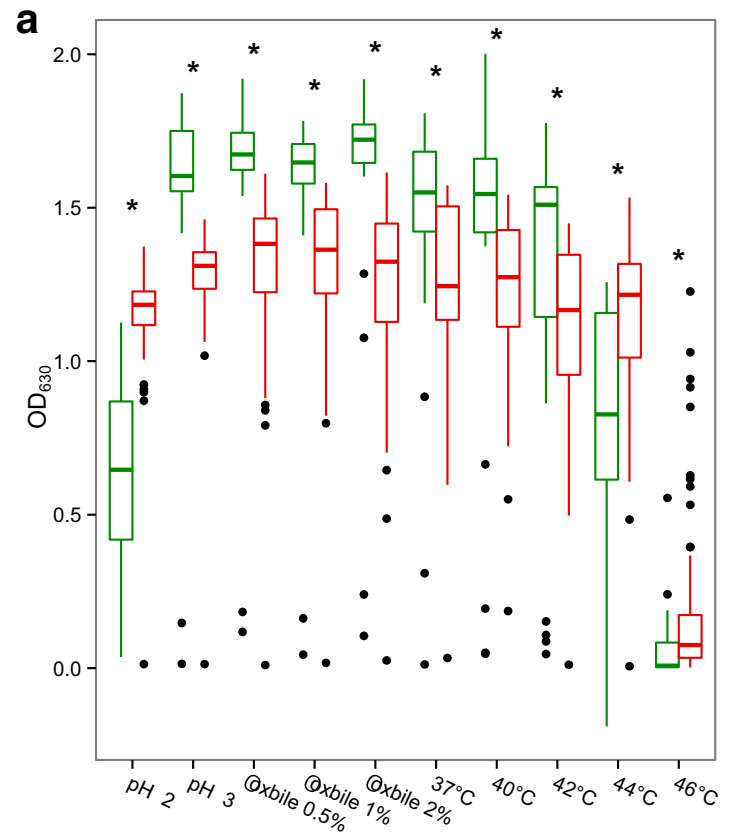

Fig. 5 In vitro tests of resistance to GI-like and stressful conditions of C. albicans isolates. a Box plot representation of the growth of different C. albicans isolated in the present study (HS, green; IBS, red), at different GI-like and stressful conditions (such as $\mathrm{pH}$, presence of bile acid, supraoptimal temperatures) as indicated by OD $630 \mathrm{~nm}$ (*FDR-corrected $p<$ 0.0005 for Wilcoxon rank-sum test). b Ability to produce hyphae or

In the gut ecosystem, fungi and bacteria coexist and directly interact with each other (Lynch and Pedersen 2016). Treatment with antibiotics in mice leads, on one hand, to the inhibition of bacteria and, on the other hand, to fungal expansion. This unbalance is then readily reverted following antibiotic cessation (Dollive et al. 2013). In an animal model of stress-induced IBS-like visceral hypersensitivity, fungicide treatment reduced visceral hypersensitivity in rat that has undergone maternal separation. Moreover, fecal transplantation in maternal separated rats showed mycobiome dysbiosis compared to non-handled rats, as controls (Botschuijver et al. 2017).

In humans, Das et al. (2021) showed that the mycobiome composition differentiates patients with IBS from controls, with a significant co-variation between mycobiota and bacteriome. Sokol et al. (2017) showed increased intermicrobial kingdom relationship in ulcerative colitis, and reduced interactions between gut bacteria and fungi in Crohn's disease, while results from Imai et al. (2019) showed the contrary. Although the results were controversial, these studies indicated that unbalanced microbial network may play a crucial role in the gut inflammation.

All the above evidences were obtained by using nextgeneration sequencing technologies that allow to explore the complexity of the gut microbial ecosystem with high resolution. In the present study, we combined culture-independent (metagenomics) and culture-based approaches (culturomics)
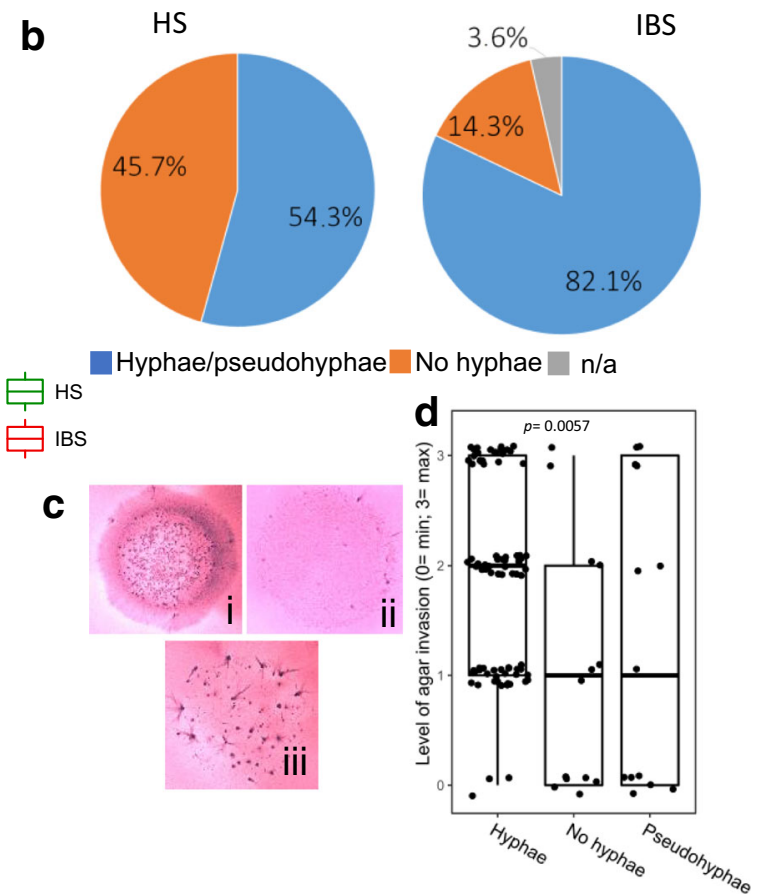

pseudohyphae. Pie charts represent the percentage of isolates able to form hyphae or pseudohyphae in both the cohorts. $\mathbf{c}$ Invasiveness test. Growth of fungal colonies in agar medium and evaluation of the different degrees of invasiveness of fungal isolates: (i) highly invasive colony; (ii) not invasive colony; (iii) minimally invasive colony. $\mathbf{d}$ Correlation between hyphal formation and invasiveness of $C$. albicans isolates

to the study of the gut fungal community. On the one hand, amplicon-based ITS1 sequencing allows for the description of fungal community composition, detecting the not-cultivable fungal population at high taxonomic level. On the other hand, culture-based analysis allows for discerning fungal phenotypes (e.g., virulence-related traits or antifungal resistance) that would be otherwise lost by metagenomics. Development of markers targeting pathogenicity traits, including markers detecting resistance to antifungals is fundamental to discriminate the "healthy" mycobiota from an altered one, often associated to disease conditions. Both methodologies are valuable, especially if combined together. In the present work, sequencing analysis showed a limited ability to identify specific taxa more than order level. We identified enrichment of Saccharomycetes in IBS compared HS. In general, fungal metagenomics procedure suffers from some limitation, especially due to the wide variance in the mycobiome taxonomic composition, as highlighted by Das et al. (2021), which suggest to consider sequencing both the ITS1 and ITS2 regions.

By using the culturomics approach, we confirmed an imbalanced fungal community in accordance with results from Gu et al. (2019) and Das et al. (2021). In both our cohorts, the most abundant fungal species was $C$. albicans, isolated at higher frequency in IBS patients than HS. This fungal species is generally recognized as human colonizer, inhabiting several body districts (Rizzetto et al. 2014). Also, Hong et al. (2020) 
found Candida as the most abundant fungal genus in IBS patients, positively correlated to severity of bloating and anxiety.

Previous evidences reported that intestinal fungal dysbiosis promote visceral hypersensitivity via the Dectin-1/Syk signaling pathway (involved in controlling of the systemic fungal infection) in IBS patients and rats (Botschuijver et al. 2017; Taylor et al. 2007). Moreover, candidalysin, a cytolytic peptide toxin produced by $C$. albicans, has been demonstrated to damage epithelial barrier and to induce mucosal inflammation (Kasper et al. 2018).

These results highlight a critical role of Candida in the pathophysiology of IBS. In our study, the phenotypical characterization of $C$. albicans isolates revealed that the isolates from IBS were significantly more resistant to in vitro stress conditions than HS ones, but they were less resistant when challenged by GI-like conditions. It is known that C. albicans changes the expression of specific $\mathrm{pH}$-related genes to inhabit tissues with different environmental $\mathrm{pH}$ (Mühlschlegel and Fonzi 1997; Calderone and Fonzi 2001) and carries out strategies to become more virulent by producing hyphae (Staib et al. 2000; Karkowska-Kuleta et al. 2009). In IBS patients, we found a high percentage of $C$. albicans isolates able to form hyphae, and to invade the agar medium, in accordance with previous observations (Felk et al. 2002). Thus, we could hypothesize that fungal isolates with peculiar virulent and invasive traits could contribute to visceral hypersensitivity associated to IBS.

Moreover, we discover differences in the genotypic background of $C$. albicans isolates between IBS and HS. Hierarchical clustering showed that IBS isolates present high genotypic relatedness among them with respect to HS isolates. We could speculate a putative clonal expansion of $C$. albicans strains in IBS, similar to our previous observations for Saccharomyces cerevisiae isolates from IBD patients (Ramazzotti et al. 2019). In this previous study, we hypothesized that in the human intestine, a clonal expansion of strains or multiple colonization of strains from the same source could occur. Both phenomena could indicate that a gut dysbiosis, occurring during inflammatory processes, could lead to the expansion or the survival of strains with peculiar characteristics contributing to gut symptoms.

In general, the specific mechanisms of the mycobiome involved in IBS yet remain to be clarified. Critical issue has been raised about the potential contribution of gut mycobiota analysis for diagnostic usefulness, although a pathogenetic importance of the mycobiota in IBS was recognized (Das et al. 2021).

Finally, we tested the different antifungal susceptibility of Candida spp. isolates (such as C. albicans, C. parapsilosis, and $C$. glabrata) from our cohorts, in order to evaluate presence of antifungal-resistant isolates in IBS. Over the last decade, the cases of infections by opportunistic pathogens, in particular of C. albicans, C. parapsilosis (Trofa et al. 2008), and C. glabrata (Fidel Jr et al. 1999), have increased greatly. It is now recognized that inappropriate antifungal use contributes to the increase in antifungal resistance, dramatically reducing the therapeutic panel and the strategies of eradication of pathogens (Sanglard and Odds 2002; Chen et al. 2010; Arendrup et al. 2011; Strati et al. 2016a). In general, our assays showed few differences in the susceptibility towards fluconazole and 5-flucytosine between Candida spp. isolates from IBS and HS. C. glabrata isolates from IBS, different from HS ones, were resistant to fluconazole, as previously described (Fidel Jr et al. 1999). C. albicans and C. parapsilosis isolates from IBS were less resistant to antifungals when compared to isolates from HS. More in general, we did not find substantial differences between IBS patients and healthy controls. However, because of increased resistance of pathogenic fungi, the classical fungicidal should be used with more caution for regular IBS therapy, although they are reserved for highly invasive and often lethal infections. Different strategies of mycobiota modulation may be appropriate for IBS, including the use of probiotics (fungal and bacterial strains) that are able to induce a favorable gut microbial immunomodulation.

Overall, our results on fungal isolate differences suggest that investigation at the strain level could be relevant for a better understanding of the role of the gut microbial community in IBS. The gut mycobiota may be involved in IBS and contribute to intestinal hypersensitivity as much as the bacterial counterpart. Future research should be addressed to investigate the potential immunomodulation of IBS fungal isolates, as previously demonstrated in IBD (Di Paola et al. 2020), and to evaluate whether bacterial and fungal dysbiosis could act in concert during the gut inflammation in IBS.

Supplementary Information The online version contains supplementary material available at https://doi.org/10.1007/s00253-021-11264-4.

Author contribution CDF, PM, FS, GMP, MDM, and MM conceived and designed the experiments. PS and FS performed the experiments. FS, PS, MDP, GA, CDF, and DC analyzed the data. PS, FS, MDP, MDV, $\mathrm{FV}$, and CDF wrote the manuscript. All authors read and approved the manuscript.

Funding Open access funding provided by Alma Mater Studiorum Università di Bologna within the CRUI-CARE Agreement. This study was funded by the University of Bologna via Grant RFO2016 2017Mattar.

\section{Declarations}

Ethics approval The study was approved by the Institutional Review Board of the Department of Gastroenterology, Piacenza Hospital (Italy). All eligible subjects provided written informed consents prior to participation in this study. 
Conflict of interest The authors declare no competing interests.

Open Access This article is licensed under a Creative Commons Attribution 4.0 International License, which permits use, sharing, adaptation, distribution and reproduction in any medium or format, as long as you give appropriate credit to the original author(s) and the source, provide a link to the Creative Commons licence, and indicate if changes were made. The images or other third party material in this article are included in the article's Creative Commons licence, unless indicated otherwise in a credit line to the material. If material is not included in the article's Creative Commons licence and your intended use is not permitted by statutory regulation or exceeds the permitted use, you will need to obtain permission directly from the copyright holder. To view a copy of this licence, visit http://creativecommons.org/licenses/by/4.0/.

\section{References}

Albanese D, Fontana P, De Filippo C, Cavalieri D, Donati C (2015) MICCA: a complete and accurate software for taxonomic profiling of metagenomic data. Sci Rep 5:9743. https://doi.org/10.1038/ srep09743

Arendrup MC, Sulim S, Holm A, Nielsen L, Nielsen SD, Knudsen JD, Drenck NE, Christensen JJ, Johansen HK (2011) Diagnostic issues, clinical characteristics, and outcomes for patients with fungemia. J Clin Microbiol 49:3300-3308. https://doi.org/10.1128/JCM.0017911

Bashir A, Miskeen AY, Hazari YM, Asrafuzzaman S, Fazili KM (2016) Fusobacterium nucleatum, inflammation, and immunity: the fire within human gut. Tumour Biol J Int Soc Oncodevelopmental Biol Med 37:2805-2810. https://doi.org/10.1007/s13277-0154724-0

Bely M, Stoeckle P, Masneuf-Pomarède I, Dubourdieu D (2008) Impact of mixed Torulaspora delbrueckii-Saccharomyces cerevisiae culture on high-sugar fermentation. Int J Food Microbiol 122:312-320. https://doi.org/10.1016/j.ijfoodmicro.2007.12.023

Benjamini Y, Hochberg Y (1995) Controlling the false discovery rate: a practical and powerful approach to multiple testing. J R Stat Soc Ser B 57:289-300

Bhattarai Y, Muniz Pedrogo DA, Kashyap PC (2017) Irritable bowel syndrome: a gut microbiota-related disorder? Am J Physiol Gastrointest Liver Physiol 312:G52-G62. https://doi.org/10.1152/ ajpgi.00338.2016

Binelli CA, Moretti ML, Assis RS, Sauaia N, Menezes PR, Ribeiro E, Geiger DCP, Mikami Y, Miyaji M, Oliveira MS, Barone AA, Levin AS (2006) Investigation of the possible association between nosocomial candiduria and candidaemia. Clin Microbiol Infect 12:538543. https://doi.org/10.1111/j.1469-0691.2006.01435.x

Botschuijver S, Roeselers G, Levin E, Jonkers DM, Welting O, Heinsbroek SEM, de Weerd HH, Boekhout T, Fornai M, Masclee AA, Schuren FHJ, de Jonge WJ, Seppen J, van den Wijngaard RM (2017) Intestinal fungal dysbiosis is associated with visceral hypersensitivity in patients with irritable bowel syndrome and rats. Gastroenterology 153:1026-1039. https://doi.org/10.1053/j.gastro. 2017.06.004

Calderone RA, Fonzi WA (2001) Virulence factors of Candida albicans. Trends Microbiol 9:327-335. https://doi.org/10.1016/s0966842x(01)02094-7

Carroll IM, Ringel-Kulka T, Siddle JP, Klaenhammer TR, Ringel Y (2012) Characterization of the fecal microbiota using highthroughput sequencing reveals a stable microbial community during storage. PLoS One 7:e46953-e46953. https://doi.org/10.1371/ journal.pone. 0046953
Castanheira M, Messer SA, Jones RN, Farrell DJ, Pfaller MA (2014) Activity of echinocandins and triazoles against a contemporary (2012) worldwide collection of yeast and moulds collected from invasive infections. Int J Antimicrob Agents 44:320-326. https:// doi.org/10.1016/j.ijantimicag.2014.06.007

Chen SC-A, Playford EG, Sorrell TC (2010) Antifungal therapy in invasive fungal infections. Curr Opin Pharmacol 10:522-530. https:// doi.org/10.1016/j.coph.2010.06.002

Chi Y, Li C, Wu L-H, Wang H-H (2020) The relationship between dectin- 1 and mast cells in patients with diarrhea-predominant irritable bowel syndrome. Scand J Gastroenterol 55:762-768. https://doi. org/10.1080/00365521.2020.1774925

Clemente JC, Ursell LK, Parfrey LW, Knight R (2012) The impact of the gut microbiota on human health: an integrative view. Cell 148(6): 1258-1270. https://doi.org/10.1016/j.cell.2012.01.035

CLSI (2017) Reference method for broth dilution antifungal susceptibility testing of yeasts. Clin Lab Stand Inst 4th Ed. CS:1-13

Collins SM (2014) A role for the gut microbiota in IBS. Nat Rev Gastroenterol Hepatol 11:497-505. https://doi.org/10.1038/ nrgastro. 2014.40

Das A, O'Herlihy E, Shanahan F, O'Toole PW, Jeffery IB (2021) The fecal mycobiome in patients with irritable bowel syndrome. Sci Rep 11:1-9. https://doi.org/10.1038/s41598-020-79478-6

DeSantis TZ, Hugenholtz P, Larsen N, Rojas M, Brodie EL, Keller K, Huber T, Dalevi D, Hu P, Andersen GL (2006) Greengenes, a chimera-checked 16S rRNA gene database and workbench compatible with ARB. Appl Environ Microbiol 72:5069-5072. https://doi. org/10.1128/AEM.03006-05

Di Paola M, Rizzetto L, Stefanini I, Vitali F, Massi-Benedetti C, Tocci N, Romani L, Ramazzotti M, Lionetti P, De Filippo C, Cavalieri D (2020) Comparative immunophenotyping of Saccharomyces cerevisiae and Candida spp. strains from Crohn's disease patients and their interactions with the gut microbiome. J Transl Autoimmun 3:100036. https://doi.org/10.1016/j.jtauto.2020.100036

Dollive S, Chen Y-Y, Grunberg S, Bittinger K, Hoffmann C, Vandivier L, Cuff C, Lewis JD, Wu GD, Bushman FD (2013) Fungi of the murine gut: episodic variation and proliferation during antibiotic treatment. PLoS One 8:e71806. https://doi.org/10.1371/journal. pone.0071806

Drossman DA (2016) Functional gastrointestinal disorders: history, pathophysiology, clinical features and Rome IV. Gastroenterology. 150: 1262-1279.e2. https://doi.org/10.1053/j.gastro.2016.02.032

Edgar RC (2004) MUSCLE: multiple sequence alignment with high accuracy and high throughput. Nucleic Acids Res 32:1792-1797

Eisenstein M (2016) Microbiome: bacterial broadband. Nature 533: S104-S106. https://doi.org/10.1038/533S104a

Englezos V, Rantsiou K, Torchio F, Rolle L, Gerbi V, Cocolin L (2015) Exploitation of the non-Saccharomyces yeast Starmerella bacillaris (synonym Candida zemplinina) in wine fermentation: physiological and molecular characterizations. Int J Food Microbiol 199:33-40. https://doi.org/10.1016/j.ijfoodmicro.2015.01.009

Felk A, Kretschmar M, Albrecht A, Schaller M, Beinhauer S, Nichterlein T, Sanglard D, Korting HC, Schäfer W, Hube B (2002) Candida albicans hyphal formation and the expression of the Efg1-regulated proteinases Sap4 to Sap6 are required for the invasion of parenchymal organs. Infect Immun 70:3689-3700. https://doi.org/10.1128/ iai.70.7.3689-3700.2002

Fidel PL Jr, Vazquez JA, Sobel JD (1999) Candida glabrata: review of epidemiology, pathogenesis, and clinical disease with comparison to C. albicans. Clin Microbiol Rev 12:80-96

Forbes JD, Bernstein CN, Tremlett H, Van Domselaar G, Knox NC (2019) A fungal world: could the gut mycobiome be involved in neurological disease? Front Microbiol 9:3249

Gayathri R, Aruna T, Malar S, Shilpa B, Dhanasekar KR (2020) Efficacy of Saccharomyces cerevisiae CNCM I-3856 as an add-on therapy 
for irritable bowel syndrome. Int J Color Dis 35:139-145. https:// doi.org/10.1007/s00384-019-03462-4

Gu Y, Zhou G, Qin X, Huang S, Wang B, Cao H (2019) The potential role of gut mycobiome in irritable bowel syndrome. Front Microbiol 10:1894. https://doi.org/10.3389/fmicb.2019.01894

Huseyin CE, O'Toole PW, Cotter PD, Scanlan PD (2017) Forgotten fungi-the gut mycobiome in human health and disease. FEMS Microbiol Rev 41:479-511. https://doi.org/10.1093/femsre/fuw047

Hong G, Li Y, Yang M, Li G, Qian W, Xiong H, Bai T, Song J, Zhang L, Hou X (2020) Gut fungal dysbiosis and altered bacterial-fungal interaction in patients with diarrhea-predominant irritable bowel syndrome: an explorative study. Neurogastroenterol Motil 32(11): e13891. https://doi.org/10.1111/nmo.13891

Iliev ID, Funari VA, Taylor KD, Nguyen Q, Reyes CN, Strom SP, Brown J, Becker CA, Fleshner PR, Dubinsky M, Rotter JI, Wang HL, McGovern DPB, Brown GD, Underhill DM (2012) Interactions between commensal fungi and the C-type lectin receptor dectin-1 influence colitis. Science (80- ) 336:1314 LP-1317 . https://doi.org/ 10.1126/science. 1221789

Imai $\mathrm{T}$, Inoue R, Kawada $\mathrm{Y}$, Morita $\mathrm{Y}$, Inatomi O, Nishida A, Bamba S, Kawahara M, Andoh A (2019) Characterization of fungal dysbiosis in Japanese patients with inflammatory bowel disease. J Gastroenterol 54:149-159. https://doi.org/10.1007/s00535-0181530-7

Ishihara S, Tada Y, Fukuba N, Oka A, Kusunoki R, Mishima Y, Oshima N, Moriyama I, Yuki T, Kawashima K, Kinoshita Y (2013) Pathogenesis of irritable bowel syndrome-review regarding associated infection and immune activation. Digestion 87:204-211. https://doi.org/10.1159/000350054

Karkowska-Kuleta J, Rapala-Kozik M, Kozik A (2009) Fungi pathogenic to humans: molecular bases of virulence of Candida albicans, Cryptococcus neoformans and Aspergillus fumigatus. Acta Biochim Pol 56:211-224

Kasper L, König A, Koenig PA, Gresnigt MS, Westman J, Drummond RA, Lionakis MS, Groß O, Ruland J, Naglik JR, Hube B (2018) The fungal peptide toxin Candidalysin activates the NLRP3 inflammasome and causes cytolysis in mononuclear phagocytes. Nat Commun 9:4260. https://doi.org/10.1038/s41467-018-06607-1

Kõljalg U, Nilsson RH, Abarenkov K, Tedersoo L, Taylor AFS, Bahram M, Bates ST, Bruns TD, Bengtsson-Palme J, Callaghan TM, Douglas B, Drenkhan T, Eberhardt U, Dueñas M, Grebenc T, Griffith GW, Hartmann M, Kirk PM, Kohout P, Larsson E, Lindahl BD, Lücking R, Martín MP, Matheny PB, Nguyen NH, Niskanen T, Oja J, Peay KG, Peintner U, Peterson M, Põldmaa K, Saag L, Saar I, Schüßler A, Scott JA, Senés C, Smith ME, Suija A, Taylor DL, Telleria MT, Weiss M, Larsson K-H (2013) Towards a unified paradigm for sequence-based identification of fungi. Mol Ecol 22:5271-5277. https://doi.org/10.1111/mec.12481

Levandowsly M, Winter D (1971) Distance between sets. Nature 234:34 35. https://doi.org/10.1038/234034a0

Li XV, Leonardi I, Iliev ID (2019) Gut mycobiota in immunity and inflammatory disease. Immunity 50:1365-1379. https://oi.org/10. 1016/j.immuni.2019.05.023

Limon JJ, Skalski JH, Underhill DM (2017) Commensal fungi in health and disease. Cell Host Microbe 22:156-165. https://doi.org/10. 1016/j.chom.2017.07.002

Longstreth GF, Thompson WG, Chey WD, Houghton LA, Mearin F, Spiller RC (2006) Functional bowel disorders. Gastroenterology 130:1480-1491. https://doi.org/10.1053/j.gastro.2005.11.061

Lynch SV, Pedersen O (2016) The human intestinal microbiome in health and disease. N Engl J Med 375:2369-2379. https://doi.org/10.1056/ NEJMra1600266

McMurdie PJ, Holmes S (2013) Phyloseq: an R package for reproducible interactive analysis and graphics of microbiome census data. PLoS One 8:e61217
Mühlschlegel FA, Fonzi WA (1997) PHR2 of Candida albicans encodes a functional homolog of the $\mathrm{pH}$-regulated gene PHR1 with an inverted pattern of $\mathrm{pH}$-dependent expression. Mol Cell Biol 17: 5960-5967. https://doi.org/10.1128/mcb.17.10.5960

Oksanen AJ, Blanchet FG, Friendly M, Kindt R, Legendre P, Mcglinn D, Minchin PR, Hara RBO, Simpson GL, Solymos P, Stevens MHH, Szoecs E (2020) Package 'vegan'. Community Ecology Package. https://cran.r-project.org, https://github.com/vegandevs/vegan

Øyri SF, Muzes G, Sipos F (2015) Dysbiotic gut microbiome: a key element of Crohn's disease. Comp Immunol Microbiol Infect Dis 43:36-49. https://doi.org/10.1016/j.cimid.2015.10.005

Pfaller MA, Diekema DJ (2012) Progress in antifungal susceptibility testing of Candida spp. by use of Clinical and Laboratory Standards Institute broth microdilution methods. J Clin Microbiol 50:2846 LP - 2856 . https://doi.org/10.1128/JCM.00937-12

Piche T (2014) Tight junctions and IBS-the link between epithelial permeability, low-grade inflammation, and symptom generation? Neurogastroenterol Motil Off J Eur Gastrointest Motil Soc 26: 296-302. https://doi.org/10.1111/nmo.12315

Pinto-Sanchez MI, Hall GB, Ghajar K, Nardelli A, Bolino C, Lau JT, Martin F-P, Cominetti O, Welsh C, Rieder A, Traynor J, Gregory C, De Palma G, Pigrau M, Ford AC, Macri J, Berger B, Bergonzelli G, Surette MG, Collins SM, Moayyedi P, Bercik P (2017) Probiotic Bifidobacterium longum NCC3001 reduces depression scores and alters brain activity: a pilot study in patients with irritable bowel syndrome. Gastroenterology 153:448-459.e8 . https://doi.org/10. 1053/j.gastro.2017.05.003

Pittayanon R, Lau JT, Yuan Y, Leontiadis GI, Tse F, Surette M, Moayyedi P (2019) Gut microbiota in patients with irritable bowel syndrome-a systematic review. Gastroenterology 157:97-108. https://doi.org/10.1053/j.gastro.2019.03.049

Plotree D, Plotgram D (1989) PHYLIP-phylogeny inference package (version 3.2). Cladistics 5:162-166

Price MN, Dehal PS, Arkin AP (2010) FastTree 2-approximately maximum-likelihood trees for large alignments. PLoS One 5:e9490

Putignani L, Del Chierico F, Vernocchi P, Cicala M, Cucchiara S, Dallapiccola B, Group DS (2016) Gut microbiota dysbiosis as risk and premorbid factors of IBD and IBS along the childhoodadulthood transition. Inflamm Bowel Dis 22:487-504. https://doi. org/10.1097/MIB.0000000000000602

Qvirist LA, Filippo CD, Strati F, Stefanini I, Sordo M, Andlid T, Felis GE, Mattarelli P, Cavalieri D (2016) Isolation, identification and characterization of yeasts from fermented goat milk of the Yaghnob Valley in Tajikistan. Front Microbiol 7. https://doi.org/ 10.3389/fmicb.2016.01690

R CoreTeam (2012) A language and environment for statistical computing. $\mathrm{R}$ foundation for statistical computing. Avaliable online at: http://wwwR-project.org/

Ramazzotti M, Stefanini I, Di Paola M, De Filippo C, Rizzetto L, Berná L, Dapporto L, Rivero D, Tocci N, Weil T, Lenucci MS, Lionetti P, Cavalieri D (2019) Population genomics reveals evolution and variation of Saccharomyces cerevisiae in the human and insects gut. Environ Microbiol 21:50-71. https://doi.org/10.1111/1462-2920. 14422

Richard ML, Lamas B, Liguori G, Hoffmann TW, Sokol H (2015) Gut fungal microbiota: the Yin and Yang of inflammatory bowel disease. Inflamm Bowel Dis 21:656-665. https://doi.org/10.1097/MIB. 0000000000000261

Rizzetto L, De Filippo C, Cavalieri D (2014) Richness and diversity of mammalian fungal communities shape innate and adaptive immunity in health and disease. Eur J Immunol 44:3166-3181. https://doi. org/10.1002/eji.201344403

Rizzetto L, Ifrim DC, Moretti S, Tocci N, Cheng S-C, Quintin J, Renga G, Oikonomou V, De Filippo C, Weil T, Blok BA, Lenucci MS, Santos MAS, Romani L, Netea MG, Cavalieri D (2016) Fungal chitin induces trained immunity in human monocytes during 
cross-talk of the host with Saccharomyces cerevisiae. J Biol Chem 291:7961-7972. https://doi.org/10.1074/jbc.M115.699645

Sanglard D, Odds FC (2002) Resistance of Candida species to antifungal agents: molecular mechanisms and clinical consequences. Lancet Infect Dis 2:73-85. https://doi.org/10.1016/s1473-3099(02)00181-0

Segata N, Izard J, Waldron L, Gevers D, Miropolsky L, Garrett WS, Huttenhower C (2011) Metagenomic biomarker discovery and explanation. Genome Biol 12:R60. https://doi.org/10.1186/gb-201112-6-r60

Simrén M, Barbara G, Flint HJ, Spiegel BMR, Spiller RC, Vanner S, Verdu EF, Whorwell PJ, Zoetendal EG (2013) Intestinal microbiota in functional bowel disorders: a Rome foundation report. Gut 62: 159-176. https://doi.org/10.1136/gutjnl-2012-302167

Sinagra E, Utzeri E, Morreale GC, Fabbri C, Pace F, Anderloni A (2020) Microbiota-gut-brain axis and its affect inflammatory bowel disease: pathophysiological concepts and insights for clinicians. World $\mathrm{J}$ Clin Cases 8:1013-1025. https://doi.org/10.12998/wjcc.v8.i6.1013

Sokol H, Leducq V, Aschard H, Pham H-P, Jegou S, Landman C, Cohen D, Liguori G, Bourrier A, Nion-Larmurier I, Cosnes J, Seksik P, Langella P, Skurnik D, Richard ML, Beaugerie L (2017) Fungal microbiota dysbiosis in IBD. Gut 66:1039-1048. https://doi.org/ 10.1136/gutjnl-2015-310746

Staib P, Kretschmar M, Nichterlein T, Hof H, Morschhäuser J (2000) Differential activation of a Candida albicans virulence gene family during infection. Proc Natl Acad Sci U S A 97:6102-6107. https:// doi.org/10.1073/pnas.110031497

Strati F, Cavalieri D, Albanese D, De Felice C, Donati C, Hayek J, Jousson O, Leoncini S, Pindo M, Renzi D, Rizzetto L, Stefanini I, Calabrò A, De Filippo C (2016a) Altered gut microbiota in Rett syndrome. Microbiome 4:41. https://doi.org/10.1186/s40168-0160185-y

Strati F, Di Paola M, Stefanini I, Albanese D, Rizzetto L, Lionetti P, Calabrò A, Jousson O, Donati C, Cavalieri D, De Filippo C (2016b) Age and gender affect the composition of fungal population of the human gastrointestinal tract. Front Microbiol 7:1227. https:// doi.org/10.3389/fmicb.2016.01227

Tap J, Derrien M, Törnblom H, Brazeilles R, Cools-Portier S, Doré J, Störsrud S, Le Nevé B, Öhman L, Simrén M (2017) Identification of an intestinal microbiota signature associated with severity of irritable bowel syndrome. Gastroenterology 152:111-123.e8 . https://doi.org/ 10.1053/j.gastro.2016.09.049

Taylor PR, Tsoni SV, Willment JA, Dennehy KM, Rosas M, Findon H, Haynes K, Steele C, Botto M, Gordon S, Brown GD (2007) Dectin1 is required for beta-glucan recognition and control of fungal infection. Nat Immunol 8:31-38. https://doi.org/10.1038/ni1408

Trofa D, Gácser A, Nosanchuk JD (2008) Candida parapsilosis, an emerging fungal pathogen. Clin Microbiol Rev 21:606-625. https://doi.org/10.1128/CMR.00013-08

Vich Vila A, Imhann F, Collij V, Jankipersadsing SA, Gurry T, Mujagic Z, Kurilshikov A, Bonder MJ, Jiang X, Tigchelaar EF, Dekens J, Peters V, Voskuil MD, Visschedijk MC, van Dullemen HM, Keszthelyi D, Swertz MA, Franke L, Alberts R, Festen EAM, Dijkstra G, Masclee AAM, Hofker MH, Xavier RJ, Alm EJ, Fu J, Wijmenga C, Jonkers DM, Zhernakova A, Weersma RK (2018) Gut microbiota composition and functional changes in inflammatory bowel disease and irritable bowel syndrome. Sci Transl Med 10: eaap8914 . https://doi.org/10.1126/scitranslmed.aap8914

Wang Q, Garrity GM, Tiedje JM, Cole JR (2007) Naive Bayesian classifier for rapid assignment of rRNA sequences into the new bacterial taxonomy. Appl Environ Microbiol 73:5261-5267. https://doi.org/ 10.1128/AEM.00062-07

Wang ZK, Yang YS, Stefka AT, Sun G, Peng LH (2014) Review article: fungal microbiota and digestive diseases. Aliment Pharmacol Ther 39:751-766. https://doi.org/10.1111/apt.12665

Publisher's note Springer Nature remains neutral with regard to jurisdictional claims in published maps and institutional affiliations. 